\title{
Prevalence of Comorbidities and Risks Associated with COVID-19 Among Black and Hispanic Populations in New York City: an Examination of the 2018 New York City Community Health Survey
}

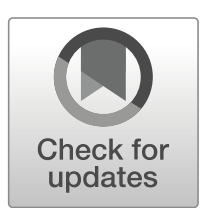

\author{
Kamyar Arasteh ${ }^{1,2}$ (D) \\ Received: 4 May 2020 / Revised: 2 August 2020 / Accepted: 4 August 2020 / Published online: 13 August 2020 \\ (C) W. Montague Cobb-NMA Health Institute 2020
}

\begin{abstract}
Background The coronavirus disease (COVID-19) pandemic has affected Blacks and Hispanics in New York City (NYC) disproportionately. This study aims to assess the relationship of race/ethnicity with COVID-19 associated factors such as hypertension, diabetes, neighborhood poverty, and frontline work, in NYC.

Methods The 2018 New York City Community Health Survey was used to examine the association of hypertension, diabetes, and neighborhood poverty level with race/ethnicity in log-binomial regression models. Number of intensive care unit (ICU) beds and the distribution of frontline workers were acquired from the US Census, the State of New York Department of Labor, and Centers for Medicare and Medicaid Services.

Results Neighborhoods with high poverty level had a greater risk of hypertension among Blacks (relative risk (RR), 95\% confidence interval (CI): 3.4, 1.9-6.4) and diabetes among Hispanics (RR, 95\% CI: 5.5, 2.2-14.0), compared with Whites in the lowest poverty neighborhoods. Disproportionately greater percentages of frontline workers were comprised of Blacks (29.4\%, 95\% CI: 29.3-29.5\%) and Hispanics (35.5\%, 95\% CI: 35.3-35.6\%). Predominantly Black and Hispanic boroughs with greater level of poverty had one ICU bed per 900 adults of 60 years of age or older, compared with one ICU bed per 452 in predominantly White boroughs with less poverty.

Conclusion The greater prevalence of the factors associated with COVID-19 infection and adverse outcomes puts Black and Hispanic populations in NYC at a greater risk. These factors are also related to poverty and should be mitigated together with reducing racial/ethnic inequities.
\end{abstract}

Keywords COVID-19 $\cdot$ Racial/Ethnic disparities $\cdot$ Hypertension $\cdot$ Diabetes

\section{Introduction}

The COVID-19 pandemic has devastated the global community. The USA has the greatest number of deaths of any country, with 64,943 at the time of this writing, on May 1, 2020 [1]. New York City (NYC) has lost 18,399 people to COVID-19, suffering the greatest burden of deaths of any city, worldwide [2]. COVID-19 deaths in NYC, as well as other metropolitan areas, have also once again shone a light on the racial/ethnic health disparities, including comorbidities associated with

Kamyar Arasteh

kamyar.arasteh@nyu.edu

1 Department of Epidemiology, School of Global Public Health, New York University, New York, NY, USA

2 Department of Epidemiology, New York University, 665 Broadway, Suite 800, New York, NY 10012, USA
COVID-19 [3]. COVID-19 hospitalizations and deaths are unevenly distributed across race and ethnicity. Non-Hispanic Blacks, $22 \%$ of the population of NYC, comprised $35 \%$ of non-fatal COVID-19 hospitalization and $31 \%$ of deaths [2]. Hispanics, $29 \%$ of the population of NYC, accounted for $31 \%$ of non-fatal hospitalization and $30 \%$ of fatalities [2]. In comparison, non-Hispanic Whites, who are $32 \%$ of the population in NYC, accounted for $24 \%$ of non-fatal hospitalization and $29 \%$ of fatalities [2]. Differences at the national level are even greater. Predominantly Black counties, for example, have three times the rate of confirmed cases of predominantly White counties [4].

Differences observed in COVID-19 hospitalization rates and mortality reflect the general trends in racial/ethnic health disparities, which arise from complex interactions of ecological variables, like poverty and access to healthcare and individual factors, such as chronic disease [5]. Poverty affects health status and survival rates. In addition, the type of labor 
force participation covaries with poverty. In NYC, $24 \%$ of frontline workers live at or below twice the poverty line [6]. Frontline workers are additionally at increased risk of COVID-19 infection due to the direct contact with COVID19 patients that may be required by healthcare workers, or from the inability to engage in social distancing, as a result of the requirements of their jobs as the police, firefighters, grocery clerks, and other essential workers. Furthermore, when it comes to the treatment of COVID-19, access to ICU beds is a key factor in the management of the severe cases of the disease [7]. Lower availability of ICU beds and ventilators in areas predominantly inhabited by communities of color would mean lower access to critical care and higher mortality.

At the individual, patient-level, the most frequently noted risk factors associated with COVID-19 hospitalization and death have been the presence of comorbidities such as hypertension $[8,9]$, diabetes [8], and coronary heart disease [10], alongside age [8]. A study of 5700 hospitalized COVID-19 patients in New York found $94 \%$ of patients had at least one comorbidity, $57 \%$ had hypertension, and $34 \%$ had diabetes [8]. By the study endpoint, $28 \%$ of hypertensive patients had died, compared with $13 \%$ of non-hypertensives. Mortality among diabetic patients was $30 \%$, compared with $18 \%$ in those without diabetes.

The purpose of the present study is to assess the association of race/ethnicity with the factors that increase the risk of adverse outcomes from COVID-19 infections in NYC. Prevalence of COVID-19 comorbidities, including hypertension and diabetes, and aggregate data with respect to the ICU beds capacity and the distribution of frontline and essential occupations are examined. Systematic, population-level assessments of COVID-19 comorbidities in the context of poverty, occupational hazard, and access to critical care associated with race/ethnicity are lacking. The study was designed to address this gap by examining these associations in Black and Hispanic populations in NYC.

\section{Methods}

Data Sources The NYC Community Health Survey (CHS) was used to obtain individual level information that are scalable to population level with respect to COVID-19 associated comorbidities. The CHS is an annual population-based survey of health information and risk behaviors of NYC residents, aged 18 and older, with a cell phone or household landline [11]. A computer-assisted telephone interview is conducted to ask participants about their cardiovascular health and diabetes, among other conditions and health-related topics. The CHS uses stratified random sampling, with stratification on the basis of age, gender, race/ethnicity, and neighborhood. The 2018 CHS was used for the present study. Aggregate data were obtained, separately, for the number of ICU beds and for the distribution of occupations across race/ethnicity. Data about the number of ICU beds in each county of NYC along with the rate of ICU beds per 100,000 people in the population of 60 years of age and older were extracted from Kaiser Health News analysis of hospital cost reports filed to the Centers for Medicare and Medicaid Services [12]. Each county of NYC was then characterized by the racial/ethnic proportions of its population, on the basis of the 2010 Census data [13]. This allowed comparing the estimates of ICU beds across counties that were comprised of predominantly Black and Hispanic residents to counties that were predominantly comprised of White residents. Additionally, detailed occupational data across race/ethnicity were obtained from the New York State Department of Labor in order to assess the distribution of race/ ethnicity across major groups of frontline workers, including healthcare workers, protective services workers, and nonprotective services workers in each county of NYC [14].

Measures Health-related measures from CHS included reported lifetime hypertension and reported lifetime diabetes. Demographic variables included race/ethnicity and age. Hispanic, Black, and White race/ethnicities were recorded as mutually exclusive categories. Age was categorized in one of the three groups of 18 to 44,45 to 64 , and 65 years of age and older. The respondent's household income level in relation to federal poverty level (FPL) and proportion of the Zip Code population living below FPL (neighborhood poverty level) were also measured. Household income level was categorized as one of the five groups of $<100 \% \mathrm{FPL}, 100$ to $<200 \% \mathrm{FPL}$, 200 to $<400 \%$ FPL, 400 to $<600 \%$ FPL, and $\geq 600 \%$ FPL. Neighborhood poverty level was categorized as percentage of population living below FPL, in one of four categories of $<$ $10 \%$ (low poverty), 10 to $<20 \%, 20$ to $<30 \%$, and 30 to $<$ $100 \%$ (high poverty). Rate of ICU beds was recorded as the inverse of the number of people 60 years of age and older per ICU bed in the county. Percentage of frontline and essential workers for each race/ethnicity was calculated as the proportion of these occupation groups across all counties of NYC who were of each race/ethnicity.

Statistical Analyses Complex survey analysis was used to accommodate the survey design utilized by CHS. All estimates from CHS data were weighted by the appropriate probability weights to represent the population of NYC. Bivariate analyses were conducted using the Pearson chi-square test, but with the test statistics corrected with Rao and Scott [15] method to account for survey design and were reported as design-based $F$ statistics, along with corresponding $p$ values. Log-binomial models were used to assess the association of risk factors with race/ethnicity in univariate and multivariable models, adjusted for gender and neighborhood poverty level, and to quantify the corresponding relative risks (RR) and $95 \%$ confidence intervals $(95 \% \mathrm{CI})$. Adjustment for gender and neighborhood 
poverty level was included because of their importance in the epidemiology and pathophysiology of disease [16-19]. When a significant interaction $(p<0.05)$ existed between covariates, RR and $95 \%$ CI of linear combinations of covariates were calculated, and the main effect of the independent variable of interest was reported at the reference level of the covariate. Separate models were fit for Blacks and Hispanics to assess the association of putative factors. Models were constructed following Agresti [20]. As age has been identified as a major risk factor for COVID-19 hospitalization and mortality and has been separately associated with hypertension [21] and diabetes [22], models were stratified by age group. Covariates with a $p$ value of less than 0.15 [23] were entered into multivariable models to adjust for their effect. Backward elimination was used to exclude covariates from the models, unless the fit of the models significantly changed. All statistical analyses were conducted using Stata, version 15 [24].

\section{Results}

CHS Data The unweighted sample consisted of 10,076 participants, representing a population of 6,670,172 adults aged 18 and older. Fifty-four percent were female (representing $3,555,781$ women in NYC population). Thirty-two percent were aged 45 to 64 (representing 2,099,488 of the NYC population in the age group), and $15 \%$ were 65 and older (representing 1,018,127 of the NYC population in the age group). Non-Hispanic Black residents comprised $22 \%$ of the population (representing 1,468,016 of the population of NYC), and Hispanics constituted 27\% (representing $1,799,268$ of the population of NYC). Table 1 shows the distribution of race/ethnicity across neighborhood poverty levels. Significantly greater percentages of Blacks $(22 \%)$ and Hispanics (29\%) lived in neighborhoods with high-poverty levels compared with Whites (5\%). The distribution of hypertension and diabetes by age group and race/ethnicity is presented in Table 2 . In every age group, reported prevalences of hypertension and diabetes were significantly greater among Blacks and Hispanics compared with Whites. Among those
65 years of age or older, $74 \%$ of Blacks and $69 \%$ of Hispanics reported hypertension, compared with $50 \%$ of Whites. In the same age group, $30 \%$ of Blacks and $39 \%$ of Hispanics reported diabetes, compared with $20 \%$ of Whites. Significantly greater proportions of Black (79\%) and Hispanic (78\%) New Yorkers aged 65 years or older reported either hypertension or diabetes compared with Whites $(56 \%)$.

Aggregated Community-Level Data In the Bronx borough of NYC, where $44 \%$ of the population are Black and $9 \%$ are nonHispanic White, the rate of laboratory confirmed cases of COVID-19 per 100,000 population was 2552 [2], and there was one ICU bed per 900 residents aged 60 or older. In contrast, in the borough of Manhattan, where $18 \%$ of the population are Black and $47 \%$ are non-Hispanic White, there were 1082 per 100,000 population (i.e., less than half of the COVID-19 rate of the Bronx) and one ICU bed per 452 residents aged 60 or older (twice the rate of ICU beds of the Bronx). Occupation data captured 640,660 frontline workers in NYC. Healthcare workers comprised $19 \%$ of the frontline and essential workers, those in protective services (such as police, firefighters, and security guards) $17 \%$, and other essential workers in non-protective services (such as mail carriers, bus drivers, and building cleaners) $64 \%$ of frontline and essential workers. Blacks were $29.4 \%$ (95\% CI: 29.3-29.5\%) and Hispanics $35.5 \%$ (95\% CI: 35.3-35.6\%) of frontline and essential workers. Whites were $20.4 \%$ (95\% CI: $20.3-20.5 \%$ ) of frontline and essential workers.

Log-Binomial Regression Models Black and Hispanic race/ ethnicity was associated with neighborhood poverty level. RR of living in the highest poverty level neighborhood (30 to $<100 \%$ FPL) was 4.0 (95\% CI: 2.6-6.3) among Blacks and 6.5 (95\% CI: 4.3-9.1) among Hispanics, compared with Whites. The RR of hypertension among Blacks living in neighborhoods with the highest poverty level, compared with Whites in the lowest-poverty neighborhoods, was 3.4 (95\% CI, 1.9-6.4). Hispanics living in neighborhoods with the highest poverty level had a RR of diabetes of 5.5 (95\% CI, 2.2-14.0), compared with Whites in the lowest poverty
Table 1 Percentage of each race/ ethnicity in 2018 CHS by neighborhood poverty level (proportion of population of the neighborhood living below FPL) across race/ethnicity in 2018 CHS

\begin{tabular}{|c|c|c|c|c|}
\hline \multirow[b]{2}{*}{$\begin{array}{l}\text { Total population } N \\
\text { Neighborhood poverty level }\end{array}$} & \multicolumn{3}{|c|}{ Race/ethnicity } & \multirow[b]{2}{*}{ Test statistic $(p)^{\mathrm{a}}$} \\
\hline & $\begin{array}{l}\text { White } \\
2,361,433 \\
\%\end{array}$ & $\begin{array}{l}\text { Black } \\
1,468,016 \\
\%\end{array}$ & $\begin{array}{l}\text { Hispanic } \\
1,799,268 \\
\%\end{array}$ & \\
\hline 0 to $<10 \%$ (low poverty) & 35 & 13 & 7 & $33(<0.0001)$ \\
\hline 10 to $<20 \%$ & 43 & 40 & 32 & \\
\hline 20 to $<30$ & 18 & 26 & 33 & \\
\hline 30 to $<100 \%$ (high poverty) & 5 & 22 & 29 & \\
\hline
\end{tabular}

${ }^{\text {a }}$ Design-corrected $F$ statistic 
Table 2 Lifetime prevalence of hypertension and diabetes reported by each age group across race/ethnicity in 2018 CHS

\begin{tabular}{|c|c|c|c|c|}
\hline \multirow[b]{2}{*}{$\begin{array}{l}\text { Total population } N \\
\text { Condition and age group }\end{array}$} & \multicolumn{3}{|c|}{ Race/ethnicity } & \multirow[b]{2}{*}{ Test statistic $(p)^{\mathrm{a}}$} \\
\hline & $\begin{array}{l}\text { White } \\
2,351,196 \\
\%\end{array}$ & $\begin{array}{l}\text { Black } \\
1,466,170 \\
\%\end{array}$ & $\begin{array}{l}\text { Hispanic } \\
1,797,232 \\
\%\end{array}$ & \\
\hline \multicolumn{5}{|l|}{ Hypertension } \\
\hline 18-44 years of age & $8.9^{\mathrm{b}}$ & 15.1 & 10.9 & $10.4(0.0001)$ \\
\hline $45-64$ years of age & 29.2 & 46.4 & 38.8 & $7.4(0.004)$ \\
\hline $65+$ years of age & 50.2 & 74.4 & 68.9 & $24.9(<0.0001)$ \\
\hline \multicolumn{5}{|l|}{ Diabetes } \\
\hline $18-44$ years of age & 1.5 & 5.0 & 4.9 & $6.1(0.004)$ \\
\hline 45-64 years of age & 7.7 & 19.4 & 21.7 & $34.6(<0.0001)$ \\
\hline $65+$ years of age & 19.7 & 30.2 & 39.1 & $15.2(<0.0001)$ \\
\hline \multicolumn{5}{|l|}{ Hypertension or diabetes } \\
\hline $18-44$ years of age & 9.7 & 16.9 & 13.8 & $11.8(<0.0001)$ \\
\hline 45-64 years of age & 31.1 & 50.1 & 45.2 & $9.7(0.0009)$ \\
\hline $65+$ years of age & 55.6 & 79.3 & 77.8 & $18.8(<0.0001)$ \\
\hline
\end{tabular}

${ }^{\text {a }}$ Design-corrected F statistic

${ }^{\mathrm{b}}$ Percentages are of each race/ethnicity within the corresponding age group neighborhoods. Table 3 presents the unadjusted RR of hypertension and diabetes by race/ethnicity within each age stratum. Unadjusted RR in all age strata of Blacks and Hispanics, except for hypertension among Hispanics 18 to 44 years of age, was significantly greater, in comparison with the Whites. Adjusted RR of hypertension and diabetes is presented in Table 4. In age-stratified multivariable models that were

Table 3 Unadjusted relative risk (RR) of hypertension and diabetes by each age group across race/ethnicity in 2018 CHS

\begin{tabular}{|c|c|c|c|c|}
\hline & \multicolumn{4}{|l|}{ Race/ethnicity } \\
\hline & \multicolumn{2}{|l|}{ Black } & \multicolumn{2}{|l|}{ Hispanic } \\
\hline & $\mathrm{RR}(95 \% \mathrm{CI})$ & $p$ & $\mathrm{RR}(95 \% \mathrm{CI})$ & $p$ \\
\hline \multicolumn{5}{|l|}{ Hypertension } \\
\hline $18-44$ years of age & $1.7(1.3-2.2)$ & $<0.001$ & $1.2(1.0-1.5)$ & 0.06 \\
\hline $45-64$ years of age & $1.6(1.2-2.2)$ & $<0.01$ & $1.3(1.1-1.6)$ & $<0.01$ \\
\hline $65+$ years of age & $1.5(1.3-1.7)$ & $<0.001$ & $1.4(1.2-1.5)$ & $<0.001$ \\
\hline \multicolumn{5}{|l|}{ Diabetes } \\
\hline $18-44$ years of age & $3.3(1.4-7.5)$ & $<0.01$ & $3.2(1.7-6.1)$ & $<0.01$ \\
\hline $45-64$ years of age & $2.5(2.0-3.2)$ & $<0.001$ & $2.8(2.1-3.7)$ & $<0.001$ \\
\hline $65+$ years of age & $1.5(1.2-2.0)$ & $<0.01$ & $2.0(1.5-2.6)$ & $<0.001$ \\
\hline \multicolumn{5}{|c|}{ Hypertension or diabetes } \\
\hline $18-44$ years of age & $1.7(1.3-2.2)$ & $<0.001$ & $1.4(1.2-1.7)$ & $<0.01$ \\
\hline $45-64$ years of age & $1.6(1.2-1.7)$ & $<0.001$ & $1.5(1.2-1.7)$ & $<0.001$ \\
\hline $65+$ years of age & $1.4(1.3-1.6)$ & $<0.001$ & $1.4(1.2-1.6)$ & $<0.001$ \\
\hline
\end{tabular}

The reference group (i.e., $R R=1.0$ ) for each $R R$ reported by race/ ethnicity within each age group is Whites of the same age adjusted for gender and neighborhood poverty level, RR of hypertension and diabetes was greater among Blacks and Hispanic across every age strata, except for hypertension among Hispanics of 45 to 64 years of age, and diabetes among Blacks and Hispanics of 65 years of age and older, compared with Whites. However, RR of diabetes in high-poverty neighborhoods among Blacks ( $R R=44.7 ; 95 \%$ CI: 8.7-228.3) and Hispanics ( $R R=48.5$; 95\% CI: 9.7-243.5) who were 65 years of age and older was much higher, in comparison with Whites.

\section{Discussion}

The findings of this study from self-reported CHS data indicate greater prevalences of hypertension and diabetes among Blacks and Hispanics, in comparison with Whites, in New York City. These results are consistent with other reports at the national level $[22,25,26]$, as well as in New York City [27]. The findings are important in and of themselves, as hypertension and diabetes are among the leading causes of morbidity and mortality in the USA [28]. Previous reports have also consistently found a higher prevalence of hypertension among Blacks, in contrast to Whites [25, 26]. Other studies have additionally found a difference in response to antihypertensive treatment [29]. However, these findings are particularly significant in the context of the association of these comorbidities with COVID-19 hospitalization and deaths. Greater rates of the COVID-19 associated comorbidities among Blacks and Hispanics put members of these race/ethnicities at a greater risk of death from a COVID-19 infection. Comorbidities in all age strata were significantly greater 
Table 4 Adjusted relative risk (RR) of hypertension and diabetes by each age group across race/ ethnicity in $2018 \mathrm{CHS}$

\begin{tabular}{|c|c|c|c|c|}
\hline & \multicolumn{4}{|l|}{ Race/ethnicity } \\
\hline & \multicolumn{2}{|l|}{ Black } & \multicolumn{2}{|l|}{ Hispanic } \\
\hline & RR $(95 \% \mathrm{CI})$ & $p$ & $\mathrm{RR}(95 \% \mathrm{CI})$ & $p$ \\
\hline \multicolumn{5}{|l|}{ Hypertension } \\
\hline $18-44$ years of age & $1.7(1.3-2.2)$ & $<0.001$ & $1.3(1.0-1.6)$ & $<0.05$ \\
\hline 45-64 years of age & $1.5(1.1-2.1)$ & $<0.05$ & $1.2(1.0-1.4)$ & 0.1 \\
\hline $65+$ years of age & $1.5(1.3-1.7)$ & $<0.001$ & $1.3(1.1-1.5)$ & $<0.01$ \\
\hline \multicolumn{5}{|l|}{ Diabetes } \\
\hline 18-44 years of age & 320.1 & $<0.001$ & 133.3 & $<0.001$ \\
\hline 45-64 years of age & $\begin{array}{l}(31.9-3217.9)^{*} \\
2.3(1.7-2.9)\end{array}$ & $<0.001$ & $\begin{array}{l}(10.3-1731.2)^{*} \\
2.3(1.7-3.2)\end{array}$ & $<0.001$ \\
\hline $65+$ years of age & $1.2(0.6-2.5)^{*}$ & 0.6 & $1.6(0.6-4.2)^{*}$ & 0.4 \\
\hline \multicolumn{5}{|c|}{ Hypertension or diabetes } \\
\hline 18-44 years of age & $2.9(1.7-5.1)^{*}$ & $<0.001$ & $1.4(1.1-1.6)$ & $<0.01$ \\
\hline $45-64$ years of age & $1.5(1.1-2.0)$ & $<0.01$ & $1.3(1.1-1.6)$ & $<0.01$ \\
\hline $65+$ years of age & $1.4(1.3-1.6)$ & $<0.001$ & $1.3(1.1-1.5)$ & $<0.001$ \\
\hline
\end{tabular}

The reference group (i.e., $R R=1.0$ ) for each $R R$ reported by race/ethnicity within each age group is Whites of the same age

*A significant interaction between race/ethnicity and neighborhood poverty level existed. The effect reported corresponds to the effect of race/ethnicity at low-poverty level neighborhood among Blacks and Hispanics. As the older population is more vulnerable to COVID-19, the greater prevalence of comorbidities among Blacks and Hispanics 65 years of age and older puts these populations at greater risk. However, even in the youngest age group (i.e., 18 to 44 years of age), prevalence of diabetes among Blacks and Hispanics was 3 times that of Whites. Therefore, the expected risk of COVID-19 infection and adverse outcomes among Blacks and Hispanics, who comprise the greatest proportion of frontline workers [6], may be increased as a result of both pre-existing comorbidities and occupational exposure.

Poverty, too, has been associated with general health [17]. When, in 2001, the state of New York expanded Medicaid to include coverage of adults with income of $100 \%$ of the federal poverty level (FPL), or less, mortality rate decreased [30]. Poverty and socioeconomic status can also influence access to critical care [31]. As patients hospitalized with acute respiratory distress may exhibit hypoxemic respiratory failure and require mechanical ventilation and extracorporeal membrane oxygenation, access to ICU beds becomes an important factor in the management of COVID-19 [7]. Although ICU capacity was greatly expanded by executive order of the governor in response to the COVID-19 epidemic in New York [32], the capacity surge is hospital-based. So, each hospital expands its capacity by the same proportion of its existing ICU beds, thereby continuing any original variability in ICU capacity.

Poverty can also affect the ability to adhere to social distancing [4, 33], which is currently the most effective prevention tool. In addition to the lower ability to socially distance in denser populations of poor neighborhoods, those in lowincome occupations, including essential workers, are less able to adhere to socially distance, in contrast to others who can work from home [33]. Hispanics and non-Hispanic Blacks in NYC are overrepresented among frontline and essential workers, including among healthcare workers, police, firefighters, grocery clerks, and others who are at a heightened risk of potential exposure to the virus. They are, therefore, at a greater risk of infection and mortality from COVID-19.

Neighborhood poverty level was also independently associated with greater risk of hypertension and diabetes in all but one age stratum. More importantly, however, neighborhood poverty compounded the relative risk of diabetes of Blacks and Hispanics 18 to 44 years of age and 65 years of age and older, compared with Whites. These observations are consistent with previous reports of the nexus of neighborhood poverty and race/ethnicity [16]. Neighborhoods with high-poverty level may contribute to the higher rates of comorbidities because healthy food may be less available [16] or a regular medical home or provider not accessible [34]. Neighborhoods with the highest poverty level also have the lowest availability of ICU beds. The NYC borough of Bronx with $58 \%$ of the population at or below "NYCgov Poverty threshold" [35] had half the rate of ICU beds per 100,000 population of adults 60 years of age and older, compared with the borough of Manhattan with $31 \%$ of the population at or below poverty threshold.

The tragedy of COVID-19 morbidity and mortality in NYC is widespread, but it is greatly exacerbated by the nexus of 
racial/ethnic health disparities, occupational hazard, and poverty. The confluence of these factors impacts the higher rates at which minority populations are exposed to COVID-19 infection, the prevalence of comorbidities that make them more vulnerable, once infected, and their access to critical care that may be necessary to combat the adverse outcomes of their COVID-19 infection that may be compounded by their comorbidities.

\section{Limitations}

This study did not assess COVID-19 infections and adverse outcomes in the same individuals who reported hypertension and diabetes. Therefore, the findings are limited to populationlevel associations. Furthermore, all the data were limited to the population of NYC. Consequently, the observations from this study may not be generalizable to other populations and geographical contexts.

\section{Conclusion}

Hypertension, diabetes, occupational exposure, and inadequate access to intensive care put Black and Hispanic populations in NYC at a greater risk of COVID-19 infection and adverse outcomes. Poverty is also related to all of these factors that are associated with the greater COVID-19 risks. To reduce racial/ethnic health inequities, all the COVID-19 associated factors must be mitigated together with poverty.

\section{Compliance with Ethical Standards}

Conflict of Interest The author declares that he has no conflict of interest.

Research Involving Human Participation This article does not contain any studies with human participants performed by the author. Retrospective data from the 2018 New York City Community Health Survey (CHS) were used.

\section{References}

1. Johns Hopkins University. JHU Covid-19 Tracker. Available from: https://coronavirus.jhu.edu/data\#charts. [Accessed: May 1, 2020].

2. New York City Department of Health and Mental Hygiene. 2020; Available from: https://www1.nyc.gov/site/doh/covid/covid-19data.page. [Accessed: May 1, 2020].

3. Laurencin CT, Mcclinton A. The COVID-19 pandemic: a call to action to identify and address racial and ethnic disparities. J Racial Ethn Health Disparities. 2020;7:398-402. https://doi.org/10.1007/ s40615-020-00756-0.

4. Yancy CW. COVID-19 and African Americans. JAMA. 2020;323: 1891. https://doi.org/10.1001/jama.2020.6548.
5. Smedley BD, Stith AY, Nelson AR, Institute of Medicine, Committee on Understanding and Eliminating Racial and Ethnic Disparities in Health Care. Unequal treatment: confronting racial and ethnic disparities in healthcare. Washington, DC: National Academies Press; 2003.

6. City of New York: Office of the Comptroller. New York City's Frontline Workers. 2020; Available from: https://comptroller.nyc. gov/wp-content/uploads/documents/Frontline_Workers_032020. pdf. [Accessed: May 1, 2020].

7. Cook DJ, Marshall JC, Fowler RA. Critical illness in patients with COVID-19: mounting an effective clinical and research response. JAMA. 2020;323(16):1559-60.

8. Richardson S, Hirsch JS, Narasimhan M, Crawford JM, McGinn T, Davidson KW, et al. Presenting characteristics, comorbidities, and outcomes among 5700 patients hospitalized with COVID-19 in the New York City area. JAMA. 2020;323:2052. https://doi.org/10. 1001/jama.2020.6775.

9. Zhou F, Yu T, du R, Fan G, Liu Y, Liu Z, et al. Clinical course and risk factors for mortality of adult inpatients with COVID-19 in Wuhan, China: a retrospective cohort study. Lancet. 2020;395(10229):1054-62.

10. Bonow RO, Fonarow GC, O'Gara PT, Yancy CW. Association of coronavirus disease 2019 (COVID-19) with myocardial injury and mortality. JAMA Cardiol. 2020;5:751. https://doi.org/10.1001/ jamacardio.2020.1105.

11. New York City Department of Health and Mental Hygiene. Community Health Survey. 2020; Available from: https://www1. nyc.gov/site/doh/data/data-sets/community-health-survey.page. [Accessed: Apr 28, 2020].

12. Schulte F, et al. Millions of older Americans live in counties with no ICU beds as pandemic intensifies. Kaiser Health News; Available from: https://khn.org/news/as-coronavirus-spreads-widelymillions-of-older-americans-live-in-counties-with-no-icu-beds/\# lookup. [Accessed: Apr 28, 2020].

13. U.S. Census Bureau. Quick Facts: New York City, New York. 2010 Census; Available from: https://www.census.gov/quickfacts/ fact/table/newyorkcitynewyork/PST045219. [Accessed: April 16, 2020].

14. New York State Department of Labor. Labor force by federal occupational classification. 2020; Available from: https://labor.ny. gov/stats/lseeo.shtm. [Accessed: Apr 28, 2020].

15. Rao JNK, Scott AJ. On chi-squared tests for multiway contingency tables with cell proportions estimated from survey data. Ann Stat. 1984;12(1):46-60.

16. Gaskin DJ, Thorpe RJ Jr, McGinty EE, Bower K, Rohde C, Young $\mathrm{JH}$, et al. Disparities in diabetes: the nexus of race, poverty, and place. Am J Public Health. 2014;104(11):2147-55.

17. Do DP, Finch BK. The link between neighborhood poverty and health: context or composition? Am J Epidemiol. 2008;168(6): 611-9.

18. Gillis EE, Sullivan JC. Sex differences in hypertension: recent advances. Hypertension. 2016;68(6):1322-7.

19. De Ritter R, et al. Sex differences in the risk of vascular disease associated with diabetes. Biol Sex Differ. 2020;11(1):1.

20. Agresti A. Categorical data analysis. 3rd ed. Wiley series in probability and statistics. Hoboken: Wiley; 2013.

21. Kearney PM, Whelton M, Reynolds K, Muntner P, Whelton PK, He J. Global burden of hypertension: analysis of worldwide data. Lancet. 2005;365(9455):217-23.

22. Selvin E, Parrinello CM, Sacks DB, Coresh J. Trends in prevalence and control of diabetes in the United States, 1988-1994 and 19992010. Ann Intern Med. 2014;160(8):517-25.

23. Hosmer DW, Lemeshow S. Applied logistic regression. New York: Wiley; 2000.

24. Statacorp, Stata Statistical Software: Release 15. 2017, StataCorp LLC: College Station. 
25. Cutler JA, Sorlie PD, Wolz M, Thom T, Fields LE, Roccella EJ. Trends in hypertension prevalence, awareness, treatment, and control rates in United States adults between 1988-1994 and 19992004. Hypertension. 2008;52(5):818-27.

26. Guo F, He D, Zhang W, Walton RG. Trends in prevalence, awareness, management, and control of hypertension among United States adults, 1999 to 2010. J Am Coll Cardiol. 2012;60(7):599606.

27. Angell SY, Garg RK, Gwynn RC, Bash L, Thorpe LE, Frieden TR. Prevalence, awareness, treatment, and predictors of control of hypertension in New York City. Circ Cardiovasc Qual Outcome. 2008;1(1):46-53.

28. Heron M. Deaths: Leading causes for 2017. National Vital Statistics Reports. 2019; 68 (6). National Center for Health Statistics: Hyattsville.

29. Lackland DT. Racial differences in hypertension: implications for high blood pressure management. Am J Med Sci. 2014;348(2): 135-8.

30. Sommers BD, Baicker K, Epstein AM. Mortality and access to care among adults after state Medicaid expansions. N Engl J Med. 2012;367(11):1025-34.
31. Lyon SM, Douglas IS, Cooke CR. Medicaid expansion under the affordable care act. Implications for insurance-related disparities in pulmonary, critical care, and sleep. Ann Am Thorac Soc. 2014;11(4):661-7.

32. New York State. Executive order No. 202.1. 2020; Available from: https://www.governor.ny.gov/news/no-2021-continuingtemporary-suspension-and-modification-laws-relating-disasteremergency. [Accessed: Apr 16, 2020].

33. Van Dorn A, Cooney RE, Sabin ML. COVID-19 exacerbating inequalities in the US. Lancet. 2020;395(10232):1243-4.

34. Huang Y, Meyer P, Jin L. Neighborhood socioeconomic characteristics, healthcare spatial access, and emergency department visits for ambulatory care sensitive conditions for elderly. Prev Med Rep. 2018;12:101-5.

35. City of New York. Poverty in NYC. 2019; Available from: https:// www1.nyc.gov/site/opportunity/poverty-in-nyc/data-tool.page. [Accessed: May 2, 2020].

Publisher's Note Springer Nature remains neutral with regard to jurisdictional claims in published maps and institutional affiliations. 